\title{
FEM_2D: A Rust Package for 2D Finite Element Method Computations with Extensive Support for hp-refinement
}

This paper was downloaded from TechRxiv (https://www.techrxiv.org).

\section{LICENSE}

CC BY 4.0

SUBMISSION DATE / POSTED DATE

$13-02-2022$ / 22-02-2022

\section{CITATION}

Corrado, Jeremiah; Harmon, Jake; Notaros, Branislav; Ilic, Milan M. (2022): FEM_2D: A Rust Package for 2D Finite Element Method Computations with Extensive Support for hp-refinement. TechRxiv. Preprint. https://doi.org/10.36227/techrxiv.19166339.v1

DOI 


\title{
FEM_2D: A Rust Package for 2D Finite Element Method Computations with Extensive Support for $h p$-refinement
}

\author{
Jeremiah Corrado* Jake J. Harmon* Milan M. Ilic* † \\ Branislav M. Notaroš*
}

14 February 2022

\begin{abstract}
FEM_2D is an open-source Finite Element Method library implemented in Rust. This MetaPaper describes some of the libraries basic functionality, as well as its unique contributions to the FEM research community. This package exhibits a powerful $h p$-refinement API with anisotropic refinements and no constraints on mesh irregularity. It also leverages Rust's Trait-Based Genericism to provide straightforward application to many problem domains. This library has been featured in some recent research in Computational Electromagnetics (see references), depicting the validity of the underlying methodology. FEM_2D has been made available as an open-source library ${ }^{1}$ with the intention of providing additional explanatory value to the existing related research, as well as to facilitate the expansion of this research area
\end{abstract}

\section{Summary}

The Finite Element Method (FEM) is a powerful tool used to solve Partial Differential Equations (PDE)s on arbitrary geometries. As its name suggests, the method works by breaking a geometric model (a Domain) into a set of small elements, each of which assigned a set of Basis Functions. A solution is expressed in terms of a weighted superposition of all the functions in the Domain such that the PDE is satisfied along with some continuity and boundary conditions. Some common applications include the Navier-Stokes equations which characterize the behavior of fluids, Schrödinger's equation which governs the evolution of quantum systems, and Maxwell's Equations which are a macroscopic description of essentially all Electromagnetic phenomena.

\footnotetext{
${ }^{*}$ Colorado State University; Department of Electrical and Computer Engineering

$\dagger$ University of Belgrade; School of Electrical Engineering

${ }^{1}$ https://github.com/jeremiah-corrado/fem_2d
} 
The FEM_2D library is a powerful FEM simulation tool implemented in Rust. It was designed within the domain of Computational Electromagnetics (CEM) with a specific focus on solving Maxwell's Equations over a 2D waveguide crosssections with very high accuracy. This problem has practical engineering applications for the design of waveguides; however, it is used here as a research tool for exploring and evaluating improvements upon FEM.

Although its initial use case was domain specific, FEM_2D's functionality extends easily to other domains using an API based on Rust Traits (which are like $\mathrm{C}++20$ Concepts or Java Interfaces). Traits are a highly expressive form of genericism which allow functions to act on any data structure so long as it implements some shared functionality. In the case of solving PDEs with FEM, that generic functionality is the ability to compute an integral over an expression of two overlapping basis functions. As such, the library's entire functionality can be leveraged against a given PDE problem by implementing FEM_2D's Integral trait to express the variational form of the problem of interest.

FEM_2D has all the functionality needed to formulate and solve Generalized Eigenvalue Problems. This includes an easy-to-use Mesh API which handles Mesh instantiation and refinement. It also includes a Domain API which defines a Generic set of Basis Functions over the Mesh while ensuring adherence to continuity and boundary conditions. Additionally, there are two EigenvalueProblem solvers. The first is implemented using Nalgebra ("Nalgebra: Linear Algebra Library for the Rust Programming Language" 2021): a powerful linear algebra library written in Rust. The second is an external sparse solver -for large or poorly conditioned problems- implemented using SLEPc ("SLEPc: Scalable Library for Eigenvalue Problem Computations" 2021) (Balay et al. 2021a) (Balay et al. 2021b) (Balay et al. 1997). The library also has extensive functionality for generating . vtk files of solutions which can be plotted using external tools.

\section{Statement of Need}

FEM_2D's primary advantage over other FEM libraries, such as the Deal.II library (Arndt et al. 2021), is its highly dynamic and expressive $h p$-refinement API. Unlike many other quadrilateral-element FEM packages, FEM_2D supports n-irregular anisotropic $h$-refinement as well as anisotropic $p$-refinement. In other words, there are far fewer limitations on the shape, location, or orientation of new elements when adding them to the Mesh. The polynomial expansion orders of the Basis Functions associated with each element can also be modified separately in each direction.

Efficiently computing solutions over geometries with sharp edges or stark material discontinuities necessitates $h p$-refinement (whether isotropic or anisotropic). These situations tend to introduce multi-scale solution behavior which is challenging to model with pure $p$ - or pure $h$-refinements, motivating combined 
hp-refinements (Harmon, Corrado, and Notaros 2021). Within the class of hp-refinements, the addition of anisotropic $h p$-refinements (over isotropic ones) presents a significantly larger capacity for solution efficiency, as small-scale behavior is targeted more directly and ineffectual Degrees of Freedom are left out of the system (Corrado, Harmon, and Notaros 2021).

The theory behind this library's $h$-refinement methodology, along with some implementation details, can be found in the associated research papers (Corrado, Harmon, and Notaros 2021), (Harmon, Corrado, and Notaros 2021).

\section{Examples of $h p$-Refinement:}

The following example shows a few of the $h p$-refinement methods on the Mesh structure and how they might be used in practice:

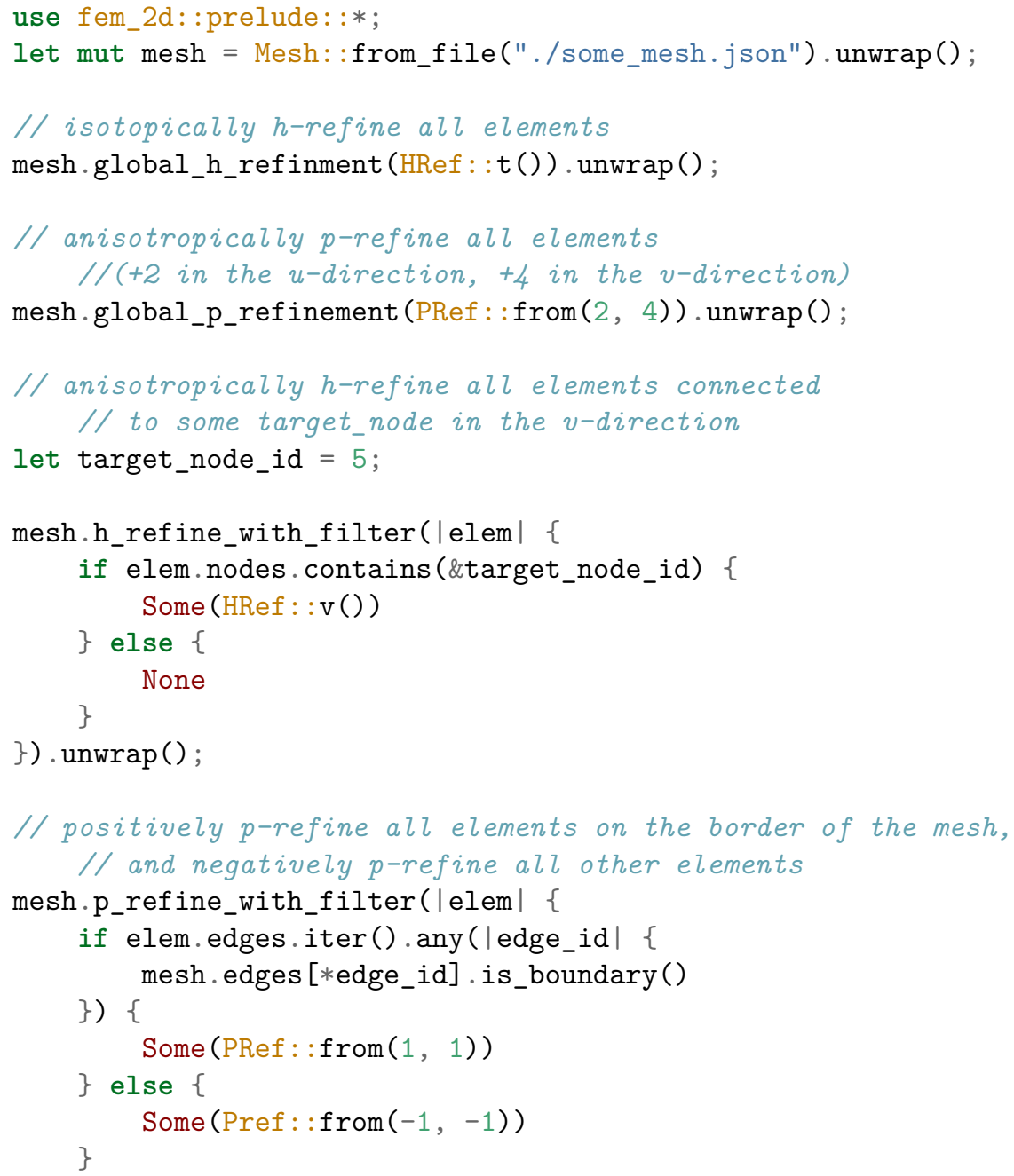


\}) $\operatorname{unwrap}()$;

\section{Example of Problem Definition}

FEM_2D also features a straightforward path to extending its functionality into other problem domains. The following example shows how a simplified formulation of the Maxwell Eigenvalue Problem maps to the corresponding code in the library. This is intended provide a general depiction of how one might translate a mathematical problem into an FEM_2D implementation.

The Maxwell eigenvalue problem has the following Continuous-Galerkin formulation for an arbitrary Domain terminated with Dirichlet boundary conditions, (constraining the solution to TE modes only):

Find a solution:

$$
\mathrm{U}=\{\mathbf{u}, \lambda\} \in B_{0} \times \mathbb{R}
$$

which satisfies:

$$
\begin{gathered}
b(\mathbf{u}, \phi)=\lambda a(\mathbf{u}, \phi) \quad \forall \phi \in B \\
\text { where: }\left\{\begin{array}{l}
B \subset H_{0}(\operatorname{curl} ; \Omega) \\
a(\mathbf{u}, \phi)=\left\langle\nabla_{t} \times \mathbf{u}, \nabla_{t} \times \phi\right\rangle \\
b(\mathbf{u}, \phi)=\langle\mathbf{u}, \phi\rangle
\end{array}\right.
\end{gathered}
$$

The system matrices for this example are populated using FEM_2D's galerkin_sample_gep method, invoked with three generic arguments:

// Generate a domain ( $\Omega$ ) from a Mesh which has been refined as necessary

let domain = Domain: :from(mesh);

// Formulate a generalized Eigenvalue problem

let gep = domain.galerkin_sample_gep: :<KOLShapeFn, CurlCurl, L2Inner $>$ (None);

The generic arguments correspond to the three lines of Equation 3

1. The curl-conforming basis $B$, which must implement the ShapeFn Trait. In this case KOLShapeFn is used.

2. The integral associated with the Stiffness Matrix (A). This argument must implement the Integral trait. In this case, CurlCurl is used.

3. The integral associated with the Mass Matrix (B). This argument also must implement the Integral trait. In this case, L2Inner is used.

The eigenvalue problem can be solved for some target eigenvalue with the following code:

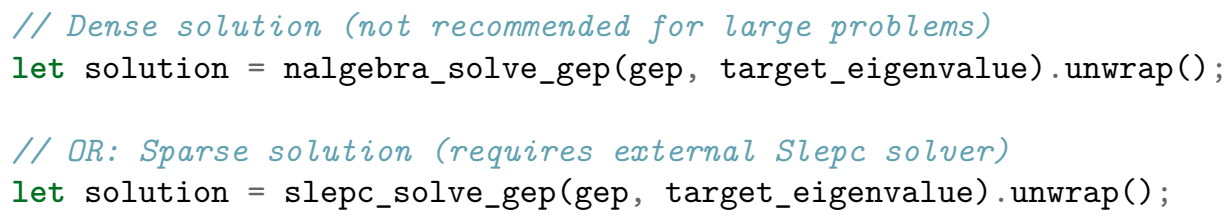


In summary, FEM_2D is useful for solving any generalized eigenvalue problem using FEM, simply by creating a custom implementation of the Integral trait. A custom Basis set can also be used by implementing the ShapeFn trait. Both Traits are described in detail in the crates.io documentation.

Additionally, the library has important ancillary functionality, such as solution plotting, and mesh plotting.

\section{References}

Arndt, Daniel, Wolfgang Bangerth, Bruno Blais, Marc Fehling, Rene Gassmöller, Timo Heister, Luca Heltai, et al. 2021. "The deal. II Library, Version 9.3." Journal of Numerical Mathematics 29 (3): 171-86. https://doi.org/10.151 5/jnma-2021-0081.

Balay, Satish, Shrirang Abhyankar, Mark F. Adams, Steven Benson, Jed Brown, Peter Brune, Kris Buschelman, et al. 2021a. "PETSc Web Page." https: //petsc.org/. https://petsc.org/.

Balay, Satish, Shrirang Abhyankar, Mark F. Adams, Steven Benson, Jed Brown, Peter Brune, Kris Buschelman, et al. 2021b. "PETSc/TAO Users Manual." ANL-21/39 - Revision 3.16. Argonne National Laboratory.

Balay, Satish, William D. Gropp, Lois Curfman McInnes, and Barry F. Smith. 1997. "Efficient Management of Parallelism in Object Oriented Numerical Software Libraries." In Modern Software Tools in Scientific Computing, edited by E. Arge, A. M. Bruaset, and H. P. Langtangen, 163-202. Birkhäuser Press.

Corrado, Jeremiah, Jake Harmon, and Branislav Notaros. 2021. "A Refinement-by-Superposition Approach to Fully Anisotropic HpRefinement for Improved Efficiency in CEM," October. https: //doi.org/10.36227/techrxiv.16695163.v1.

Harmon, Jake, Jeremiah Corrado, and Branislav Notaros. 2021. "A Refinementby-Superposition Hp-Method for $\mathrm{h}$ (curl)- and $\mathrm{h}$ (div)-Conforming Discretizations," June. https://doi.org/10.36227/techrxiv.14807895.v1.

"Nalgebra: Linear Algebra Library for the Rust Programming Language." 2021. GitHub Repository. GitHub. https://github.com/dimforge/nalgebra.

"SLEPc: Scalable Library for Eigenvalue Problem Computations." 2021. GitHub Repository. GitHub. https://github.com/firedrakeproject/slepc. 